\title{
EDIFICIOS CON FORJADOS POSTESADOS MEDIANTE TENDONES NO ADHERENTES. EDIFICIO MAPFRE-VÍA AUGUSTA, EN BARCELONA-ESPAÑA
}

\author{
(BUILDINGS WITH SLAB FLOORS POST-TENSIONED BY UNBONDED TENDONS)
}

José Antonio Llombart Jaques, Ingeniero de Caminos

Jordi Revoltós Fort, Ingeniero de Caminos

Fecha de recepción: 12 - VIII - 94

Estudio de Ingeniería y Proyectos (EIPSA), Madrid

\section{RESUMEN}

La técnica del postesado no adherente, aplicada a losas de forjado de edificios de cierta entidad, ba sido hasta el momento poco común en España, habiéndose utilizado solamente en contadas ocasiones.

Se indican, en primer lugar, las características fundamentales de los forjados postesados y se establecen comparaciones con soluciones convencionales de forjados de bormigón armado.

En la segunda parte del artículo se describen los principales aspectos de proyecto y construcción de la estructura del Edificio Mapfre-Vía Augusta, en Barcelona, de reciente construcción, cuyos forjados están constituidos por losas postesadas mediante tendones no adberentes.

\section{SUMMARY}

The technique of unbonded tendons applied to post-tensioned flat-slabs of buildings of certain importance has not been very frequent in Spain to date; in fact, it was used on very few occasions.

This article gives the basic characteristics of the post-tensioned floors comparing them with the conventional solutions of reinforced concrete floors.

The second part of the article brings a description of the main aspects of the design and construction of the Mapfre-Via Augusta building in Barcelona. This recently raised building has the floor construction with slabs post-tensioned by unbonded tendons.

\section{Edificios con forjados postesados mediante tendones no adherentes}

\subsection{Introducción}

La aplicación estructural del postesado no adherente ha constituido durante los últimos años, en Estados Unidos, una técnica usual para la construcción de edificios de hormigón (conjuntos residenciales, edificios de oficinas, aparcamientos, hospitales, edificios industriales, etc.), debido a sus favorables aspectos económicos, fiabilidad, durabili- dad, posibilidad de lograr espacios de gran diafanidad, constitución de losas de espesor uniforme y de gran esbeltez, eliminación de problemas derivados de la flexibilidad de forjados, posibilidad de construcción acelerada, por el hecho de desencofrar los forjados al finalizar la puesta en tensión de las armaduras activas, etc.

La técnica del postesado no adherente se ha extendido en númerosos países de todo el mundo. En Europa ha tenido una notable difusión, especialmente en Suiza e Inglaterra, sin embargo, sus apli- 
caciones en España han sido escasísimas hasta el actual momento, a pesar del magnífico resultado obtenido en recientes realizaciones, una de las cuales se muestra en el presente artículo.

A continuación, se exponen algunas de las características más importantes de los forjados resueltos con losas de hormigón postesadas mediante tendones no adherentes $y$, finalmente, se describen los principales aspectos de proyecto y construcción de la estructura del Edificio Mapfre-Vía Augusta, en Barcelona.

\subsection{Losas de forjado postesadas y sus ventajas fundamentales en relación con los forjados simplemente armados}

En las piezas de hormigón armado, tales como las losas de forjado, la fisuración del material debida a la existencia de tensiones de tracción concentradas en las armaduras es una consecuencia natural de los estados de flexión inducidos por las cargas verticales y de la propia naturaleza del hormigón.

En las losas postesadas, si bien no se eliminan en su totalidad las tensiones de tracción, quedan notablemente reducidas y, por tanto, la fisuración. Ello trae como consecuencia un aumento de la resistencia global, frente a la flexión y esfuerzo cortante, junto con un aumento de la rigidez, por lo que el espesor de las piezas puede ser menor que en las losas simplemente armadas.

A ello hay que añadir el efecto causado por el trazado curvo de los tendones y su disposición excéntrica en relación con el centro de gravedad de la losa. Las fuerzas de desviación, debidas al pretensado, actúan en dirección opuesta a las cargas verticales de peso propio, de tal forma que en la práctica pueden llegar a neutralizarse mutuamente, a efectos de la flecha resultante en el centro de los vanos. Consecuentemente, la flecha vertical diferida motivada por la fluencia queda totalmente eliminada.

En resumen, las ventajas fundamentales de tipo estructural pueden resumirse en los siguientes conceptos: a) aumento de la resistencia; b) aumento de la rigidez; y c) disminución o eliminación de la flecha vertical, instantánea y diferida debido a la acción de las cargas muertas. Esta última característica tiene una gran importancia en el proyecto de los edificios, por lo que estimamos conveniente dedicar un capítulo a continuación.

\subsection{Flexibilidad de los forjados}

Los requisitos funcionales plasmados en los proyectos de Arquitectura, junto con la necesidad de máximo aprovechamiento de los espacios disponibles para la construcción de edificios exigen, cada vez más, reducir el espesor de los forjados y evitar la disposición de vigas descolgadas.

La consideración de la seguridad estructural no es suficiente para determinar el espesor óptimo de los forjados de hormigón armado desde un punto de vista funcional. La limitación de la flexibilidad suele representar el aspecto crítico que condiciona, en la mayor parte de las ocasiones, la magnitud del espesor de la estructura que constituyen los entrepisos.

En las piezas de hormigón armado, la determinación de la flexibilidad y, por tanto, la cuantificación de la deflexión en el centro de los vanos debe hacerse teniendo en cuenta necesariamente la circunstancia de la fisuración como pieza flectada. La fisuración reduce notablemente la rigidez, cuyo valor depende de la inercia fisurada, mucho menor que la que se deduciría geométricamente de la sección bruta. El cálculo de la flecha, en cualquier caso, sólo puede realizarse de una forma aproximada debido a la incertidumbre existente en el conocimiento de la fisuración, especialmente si se trata de piezas de una cierta complejidad estructural, cuyo esquema se aparta del de las vigas simplemente apoyadas, para las que existen fórmulas que permiten determinar las flechas con razonable aproximación.

Las cargas permanentes debidas a la acción conjunta del peso propio más la carga muerta de solados, rellenos y tabiques producen un incremento gradual de la flecha instantánea, debido a la fluencia, de tal forma que la deformación total, incluida la adicional diferida, resulta ser del orden de 3,5 veces la instantánea en climas secos y 2,5 veces en climas húmedos.

En los forjados de edificación, normalmente el valor de las cargas permanentes (peso propio más carga muerta), suele ser superior al de las sobrecargas de uso. Por tal motivo, la consideración de los criterios antedichos, aplicados al cálculo de la flecha total debida a la carga permanente, son determinantes para la elección del espesor de los forjados al objeto del cumplimiento de Normas, por una parte, y para evitar, además, el riesgo de patología y grietas en la tabiquería, que a menudo suelen producirse durante los años siguientes a la entrada en ser- 
vicio de edificios con forjados sin vigas de una determinada esbeltez.

En el caso de forjados postesados, la consideración de la flexibilidad requiere un tratamiento totalmente distinto, ya que existen tensiones de compresión en la totalidad de la pieza bajo la acción de cargas permanentes. La inercia de la sección (inercia bruta) deducida directamente de las dimensiones exteriores, puede ser aplicada en los cálculos de la flecha sin descontar la zona fisurada. La rigidez, y por tanto la flecha, puede ser determinada con razonable precisión, conociendo el módulo de deformación del hormigón.

Además de la ventaja relativa a la rigidez, mucho mayor en un forjado postesado que en el armado, debe añadirse la característica fundamental y más importante, derivada de las fuerzas de desviación del postesado: la flecha debida a las cargas permanentes puede reducirse o anularse por completo, en función del diseño estructural efectuado por el Proyectista.

En resumen, en un forjado postesado, sea cual fuere el espesor, puede proyectarse la estructura de forma que, bajo la acción de las cargas permanentes, la flecha instantánea y la diferida sean nulas. Unicamente deberá valorarse la flecha debida a las sobrecargas que, en cualquier caso, será mucho menor que la que resultaría en un forjado de hormigón armado de idénticas dimensiones exteriores.

Por tal motivo la consideración de la flexibilidad no suele resultar crítica en los proyectos de edificios con forjados postesados, y de ahí una de las ventajas funcionales de mayor importancia.

\subsection{Posibilidades de diseño}

El empleo de postesado no adherente ofrece magníficos recursos de diseño estructural, aportando ventajas en el proyecto general de Arquitectura, en relación con soluciones convencionales de hormigón armado, que suelen hacerse patentes cuando se trata de conseguir grandes espacios diáfanos, soportar fuertes sobrecargas de uso y conseguir al mismo tiempo reducidos espesores de forjado, que pueden optimizarse mediante la disposición de capiteles o ábacos en la coronación de pilares. Las limitaciones en el diseño suelen responder normalmente a motivos económicos o requisitos derivados de resistencia estructural, pudiéndose disponer en los casos normales y sin grandes problemas relaciones de espesor/luz comprendidas entre $1 / 30$ y $1 / 40$.
Las posibilidades aportadas por la tecnología del postesado no adherente aplicadas a la edificación exigen, al mismo tiempo, una cuidadosa puesta a punto de los sistemas de diseño y análisis estructural, especialmente cuando se trata de edificios con disposiciones de pilares poco moduladas, peculiaridades motivadas por los huecos de instalaciones, escaleras, etc.; todo ello de gran importancia para trazar adecuadamente los tendones de pretensado $\mathrm{y}$, por tanto, las fuerzas de desviación que deben estar dimensionadas y ajustadas de acuerdo con las características geométricas del forjado. Contrariamente a lo que ocurre con las armaduras pasivas, un exceso en la armadura activa, así como en las fuerzas de pretensado, podría acarrear serias consecuencias negativas de tipo estructural.

\subsection{Construcción}

En lo referente a la construcción, los forjados postesados aportan una notable ventaja en lo relativo a la rapidez de puesta en obra. El descimbrado de las losas, consecuencia del tesado de tendones, puede realizarse a muy temprana edad del hormigón, por lo cual, el avance de la obra no está tan condicionado como en los forjados de hormigón armado.

La colocación de los tendones de postesado y su correcto posicionado implican a menudo un esfuerzo de organización para el constructor y una cuidadosa planificación de actividades, en la que difícilmente cabe la improvisación. El ajuste de la obra a las especificaciones requiere mayores exigencias, si cabe, que en los forjados de hormigón armado, a causa de la repercusión que cualquier modificación podría tener en las fuerzas debidas al pretensado.

\section{Edificio Mapfre-Via Augusta, en Barcelona}

\subsection{Descripción y dimensiones fundamentales}

El Edificio Mapfre-Vía Augusta, de Barcelona, está situado en un solar, limitado por la Vía Augusta y las calles General Mitre y Ganduxer (Fig. 1).

El edificio dispone de cinco plantas subterráneas destinadas a aparcamiento. Sobre el nivel de planta baja se levantan dos torres gemelas destinadas a locales comerciales y oficinas (Fig. 2). Los forjados de las torres, cada uno con una superficie aproximada de $1.400 \mathrm{~m}^{2}$, están constituidos por losas aligeradas, habiéndose dispuesto postesado mediante tendones no adherentes. 


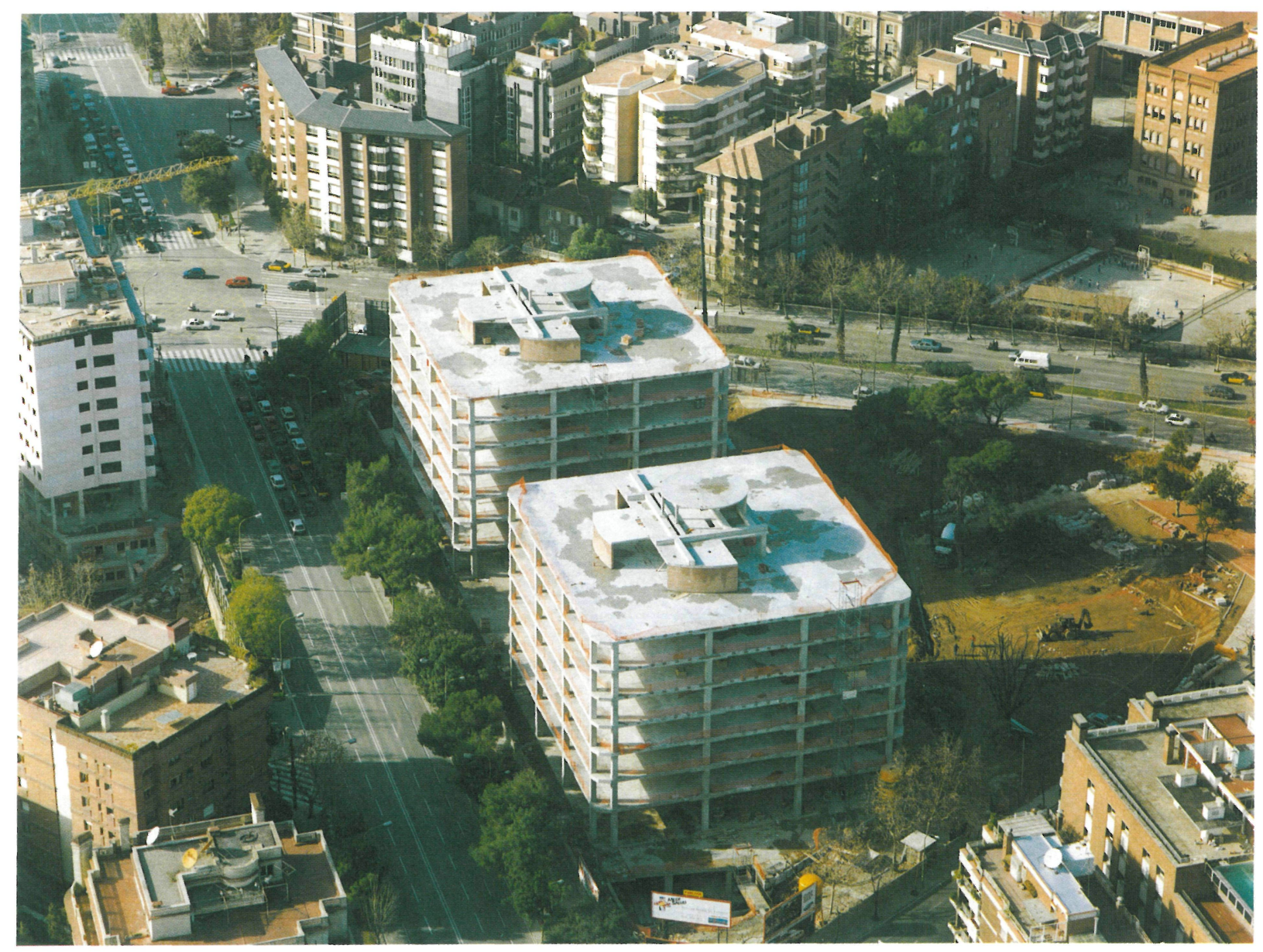

Fig. 1. Vista general del Edificio Mapfre-Vía Augusta, en Barcelona, en la fase de terminación de la estructura.

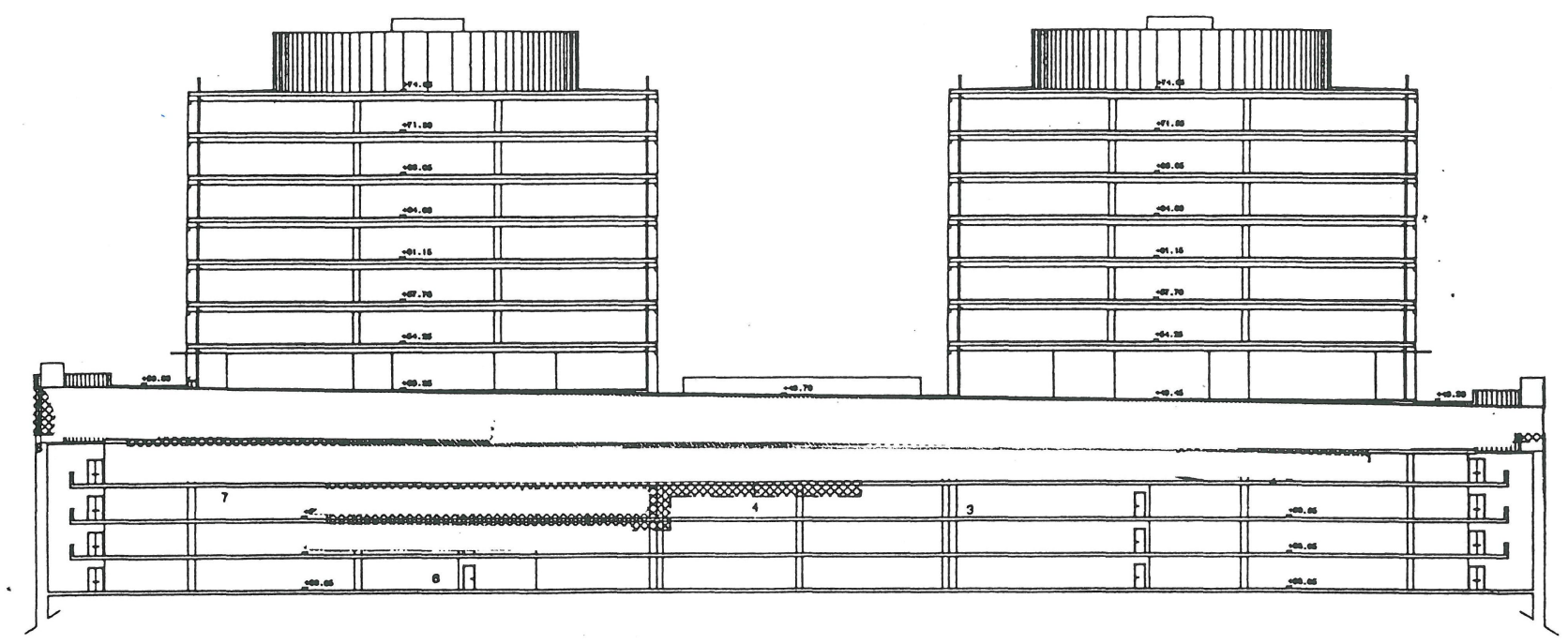

Fig. 2. Estructura del edificio. Sección. 

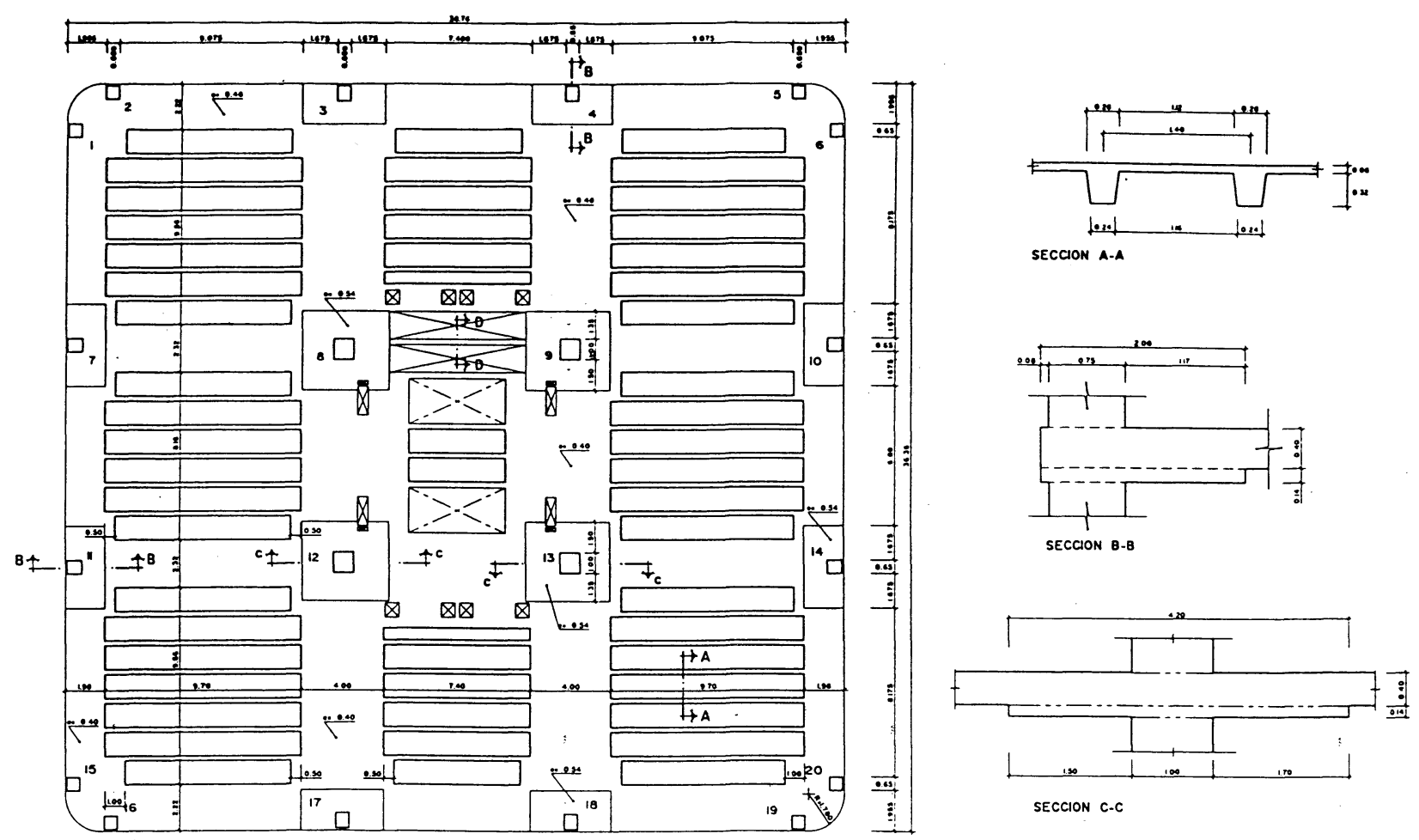

Fig. 3. Planta tipo. Definición geométrica.

Existen, en total, ocho forjados postesados en cada una de las torres, cuyas dimensiones en planta son 38,76 x 36,36 m. La separación de pilares, con luces máximas de 13,35 m entre ejes y la limitación del espesor de los forjados $(40 \mathrm{~cm})$, han motivado la resolución de la estructura resistente mediante el sistema que se describe a continuación.

\subsection{Planteamiento general del proyecto de la estructura}

En el edificio Mapfre concurre una serie de exigencias funcionales, derivadas del proyecto general de Arquitectura, que han sido determinantes para la adopción del tipo de estructura llevada a la práctica. He aquí un resumen de los aspectos de mayor importancia tenidos en cuenta en el proyecto:

- Creación de espacios de gran diafanidad en las plantas de las dos torres gemelas. Necesidad de resolver forjados con separación entre ejes de pilares, dispuestos ortogonalmente, de 13,35 x 12,45 m. Los vanos de máxima luz se encuentran en las zonas del edificio más próximas a las fachadas.
- Limitación del espesor del forjado, al objeto de disponer del máximo número de plantas, de forma compatible con la altura del edificio, limitada por las Ordenanzas. Disposición de forjados, sin vigas, con un espesor de $40 \mathrm{~cm}$.

- Sobrecargas de uso: $700 \mathrm{~kg} / \mathrm{m}^{2}$ en planta baja, apta para locales comerciales, $400 \mathrm{~kg} / \mathrm{m}^{2}$ en plantas de oficinas y $1.000 \mathrm{~kg} / \mathrm{m}^{2}$ en gran parte de la planta de cubierta, para montaje de instalaciones.

- Cargas muertas en el borde exterior de los forjados debido al peso propio de las fachadas constituidas por elementos prefabricados.

- Disposición de grandes huecos en la superficie de forjados para paso de escaleras de servicio, ascensores, escaleras mecánicas y conducciones para instalaciones. Algunos de los mencionados huecos están situados en las inmediaciones de los pilares más solicitados por cargas verticales (Fig. 3).

Además de las exigencias resistentes de la estructura, deben añadirse los requisitos referentes a la 
limitación de la flexibilidad de los forjados, preceptiva para toda obra de edificación.

Una vez analizado el conjunto de condicionantes, se decidió constituir los forjados mediante losas aligeradas de hormigón, provistas con postesado no adherente, considerándose dicha solución como la única que permitía resolver la estructura de una forma técnicamente satisfactoria.

\subsection{Tipología estructural del conjunto}

Sobre el nivel de rasante, el edificio está constituido por dos torres independientes, cuyos elementos verticales de soporte arrancan de la zona de sótano. En cada una de las dos torres, existe un total de ocho plantas, caracterizadas por la diafanidad interior y la distancia existente entre ejes de pilares.

La magnitud de las cargas verticales que actúan sobre los pilares que soportan la torre, han determinado sus dimensiones transversales aparentemente robustas, si se considera únicamente que se trata de un edificio con ocho forjados sobre rasante. Sin embargo, si se tiene en cuenta que el peso propio de un forjado postesado es notablemente superior al de un forjado reticular de luces medias y que la separación entre ejes de pilares en las dos dimensiones perpendiculares representa el doble de las habituales en otro tipo de edificios, puede deducirse fácilmente que la magnitud de la carga vertical, por pilar, es del orden de seis o siete veces la que resultaría en el caso de haberse dispuesto una estructura convencional. Por tal motivo, los pilares principales del Edificio Mapfre están capacitados para soportar una carga vertical propia de un edificio de 50 plantas. La carga vertical de servicio de dichos pilares, a nivel de cimentación, alcanza un valor de 2.200 toneladas.

Las obligadas dimensiones transversales de los pilares por motivos resistentes traen consigo la consecuencia de poseer una gran rigidez, y tal consideración ha representado una de las mayores dificultades para resolver adecuadamente el esquema estructural de las dos torres.

La coacción ejercida por los pilares frente al giro y al desplazamiento horizontal suele ser despreciable en la mayor parte de los edificios resueltos con forjados postesados; la magnitud del acortamiento elástico producido por la acción del pretensado es compatible con la flexibilidad de los pilares, sin representar esfuerzos parásitos de consideración. Sin embargo, en el Edificio Mapfre, el efecto causado por la rigidez de los pilares tiene gran importancia, y por tal motivo ha sido objeto de consideración rigurosa en el cálculo estructural y al mismo tiempo ha precisado de una especial disposición en la planta baja, más influenciada por tal efecto: dicho forjado no está unido monolíticamente a los pilares inferiores del contorno, habiéndose dispuesto apoyos intermedios de neopreno que permiten liberar, tanto el giro, como el desplazamiento horizontal (Fig. 4).

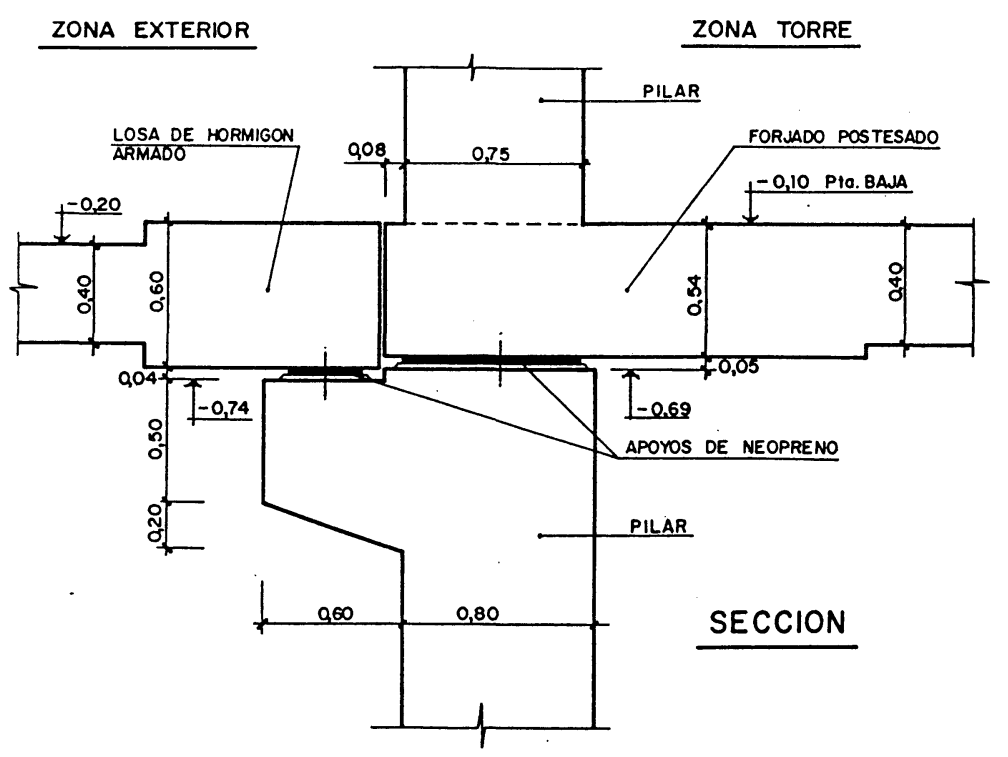

Fig. 4. Detalle de la zona de apoyo de pilares de planta baja. 
La rigidez y estabilidad del conjunto de cada una de las torres frente a la acción de cargas horizontales no resultan afectadas por tal disposición, debido a la existencia de unos elementos apantallados dispuestos en el núcleo de ascensores y que, partiendo de la cimentación, quedan interrumpidos a nivel del forjado de la planta primera. A partir de dicha planta, todos los pilares están rígidamente empotrados con las losas de forjado y constituyen en su conjunto un adecuado sistema estructural en lo referente a estabilidad frente a acciones horizontales.

Las dimensiones transversales de los pilares están limitadas inferiormente por condicionantes de punzonamiento de los forjados que soportan, y superiormente para evitar un exceso de rigidez. Por tal motivo, la escuadría de los pilares permanece constante a lo largo de toda la altura de las torres, al objeto de mantener la superficie de apoyo en el contacto con las losas y, por tanto, la seguridad frente al punzonamiento. Dicha condición alcanza su máxima exigencia en el forjado de la última planta, cuya sobrecarga de uso es superior a la del resto de los forjados.

Con objeto de mejorar la seguridad frente al punzonamiento, se han dispuesto ábacos sobre los pilares más solicitados, que constituyen un descuelgue de 14 centímetros, en relación con el paramento inferior de los forjados (Fig. 5).

\subsection{Postesado no adherente. Disposición y caracteristicas}

El postesado se ha realizado mediante tendones constituidos, cada uno, por un cordón de 0,6", engrasado y envuelto en vaina de polietileno. Los

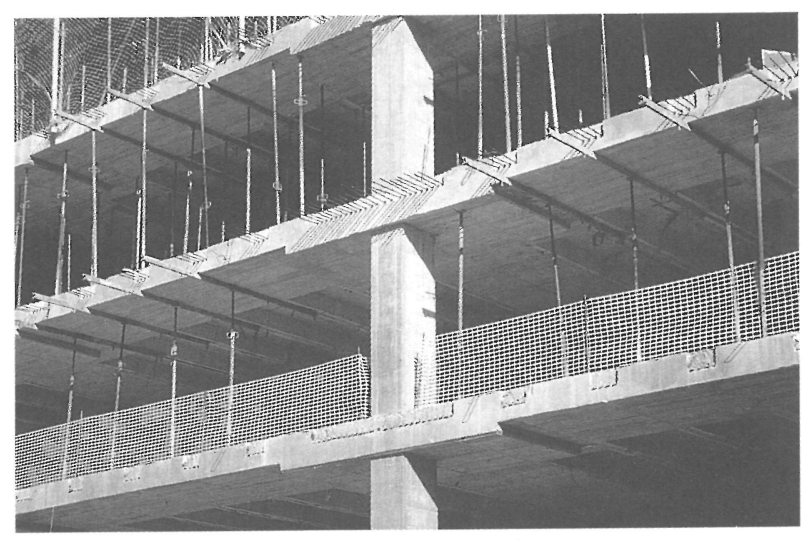

Fig. 5. Borde del edificio, en construcción. Se aprecian los anclajes de postesado no adberente.

elementos de anclaje son independientes para cada tendón, habiéndose dispuesto anclajes activos, únicamente en el contorno exterior del forjado (Fig. 6). Existen anclajes pasivos en el interior de las losas, en las inmediaciones de los pilares centrales (Fig. 7), así como en los paramentos de huecos intermedios. En este último caso, se trata de anclajes idénticos a los activos situados en el contorno, si bien no se ejerce tesado por el extremo interior.

La mayor parte del acero activo se encuentra concentrada en unas franjas en que se ha macizado la losa y cuyas alineaciones en las dos direcciones perpendiculares están determinadas por la posición de los pilares principales (Figs. 8 y 9). En las zonas intermedias limitadas por dichas franjas se ha distribuido uniformemente un postesado secundario unidireccional, que discurre por los nervios situados entre los aligeramientos. Esta disposición permite resolver fácilmente el cruce con los tendones de las calles principales (Figs. 10 y 11).

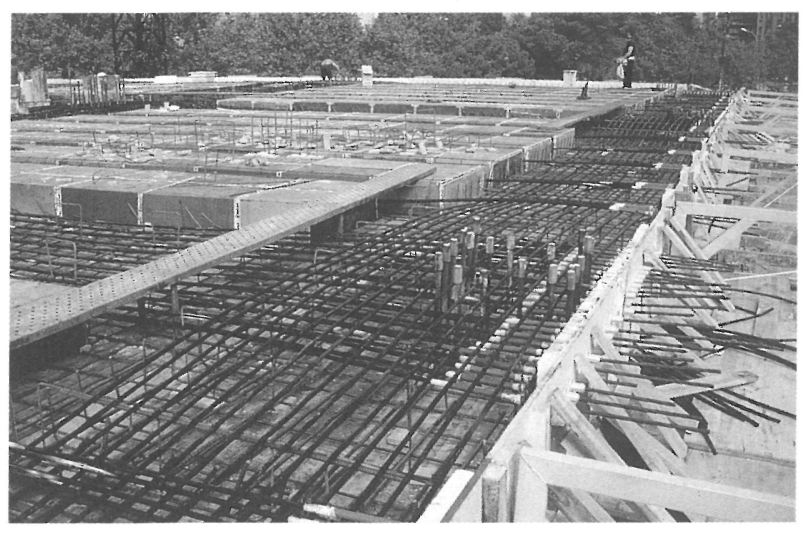

Fig. 6.

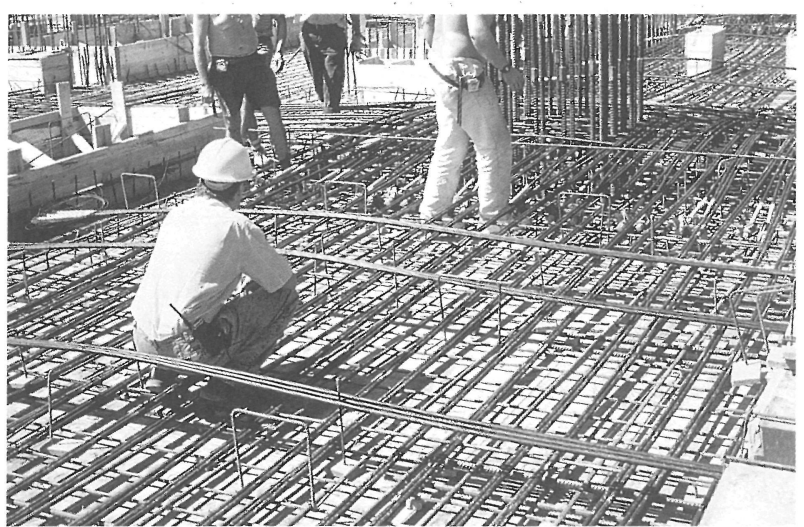

Fig. 7. 

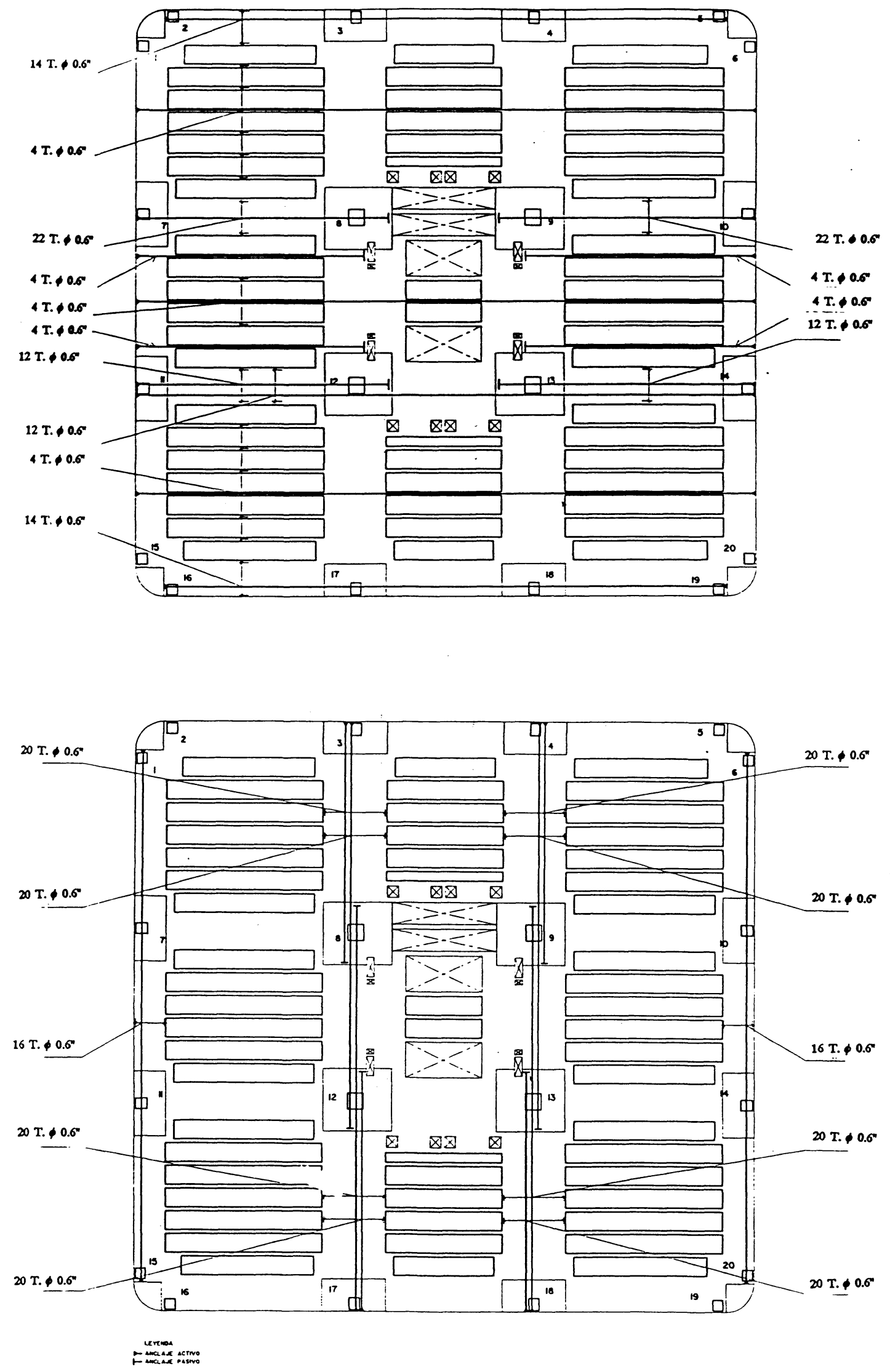

Fig. 8. Planta tipo. Trazado de pretesado en "X" y en " $Y$ ". 


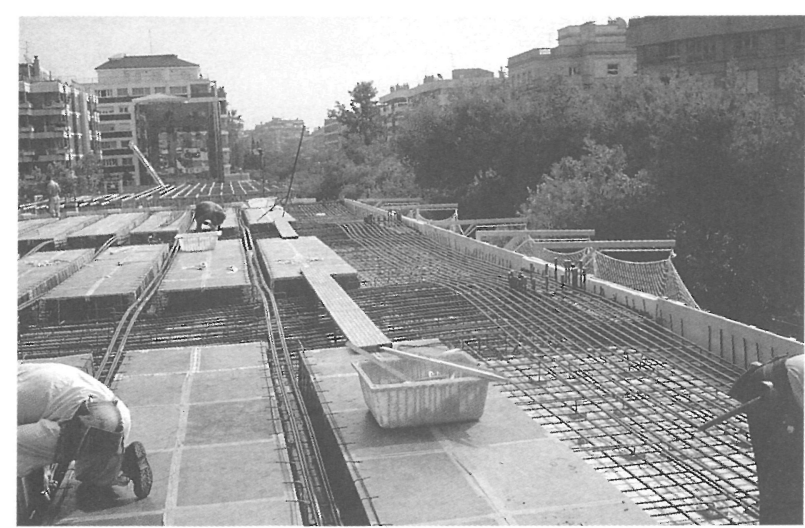

Fig. 9.

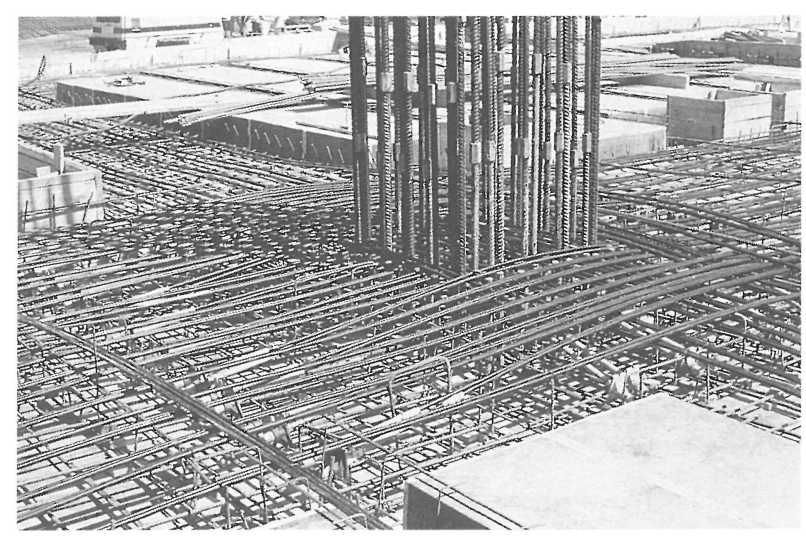

Fig. 12. Detalle de postesado en zona de arranque de pilares

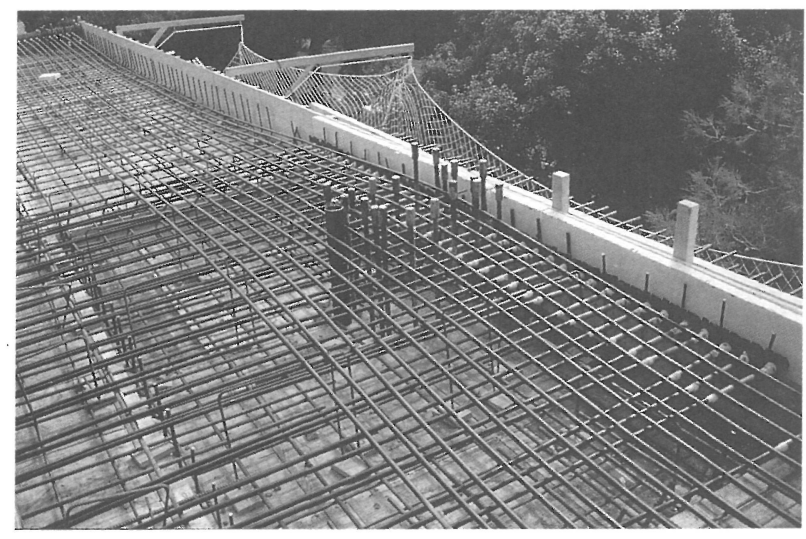

Fig. 10.

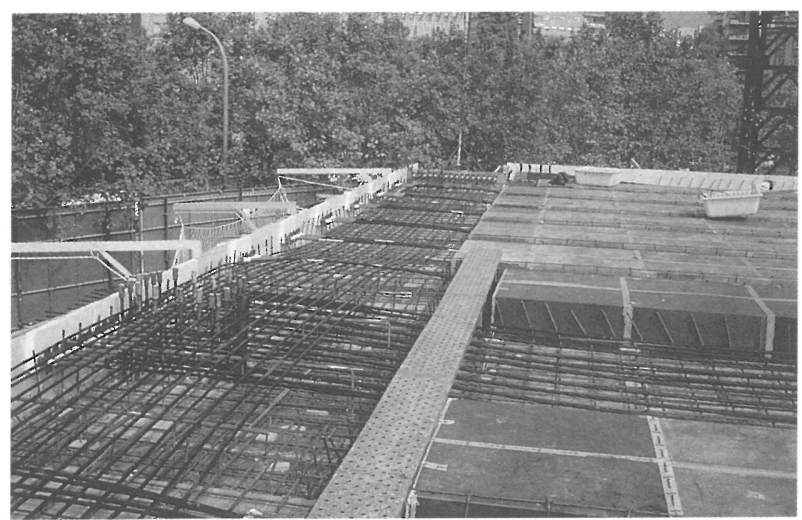

Fig. 11.

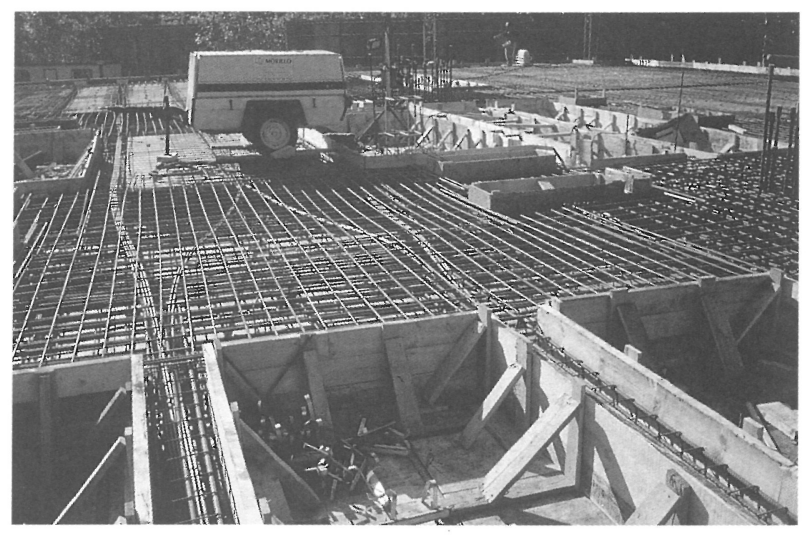

Fig. 13. Tendones de postesado en los bordes de grandes buecos.

La necesaria concentración de armadura activa en las zonas situadas sobre los pilares (Fig. 12) y en los bordes de los grandes huecos (Fig. 13), exigió un detallado estudio, tanto a nivel del proyecto, por motivos de orden estructural y de encaje geométrico, como durante la ejecución de la obra, habiendo sido necesario planificar con todo detalle el orden de colocación de los distintos tendones. La colocación de los tendones se llevó a cabo cumpliéndose unos estrictos criterios de tolerancias en la posición y en el trazado, debido a la influencia que podrían causar posibles desviaciones que, aun siendo pequeñas en valor absoluto, podrían tener importancia dado el reducido canto de las losas. Asimismo se consideró de gran importancia la limitación de la distancia comprendida entre los puntos de fijación de los tendones, cuyo valor máximo 


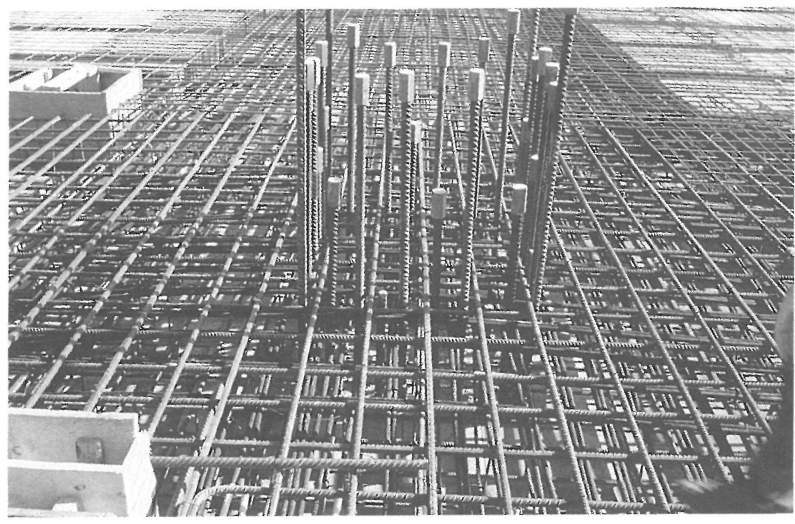

Fig. 14 .

figuró como una especificación del proyecto a fin de evitar el "efecto guirnalda", cuya consecuencia podría haberse manifestado en un aumento de las pérdidas de rozamiento previstas en el proyecto.

El efecto del pretensado dispuesto compensaba ligeramente las cargas debidas al peso propio, de tal forma que una vez finalizadas las operaciones de tesado, el forjado quedaba ligeramente despegado de la cimbra en el centro de los vanos; $3 \mathrm{~mm}$ aproximadamente. Posteriormente, y para poder soportar el peso propio del forjado superior en proceso de hormigonado, fue preciso proceder al recimbrado, dado el considerable peso propio, que superaba el valor de las sobrecargas de uso previstas en el cálculo.

\subsection{Análisis estructural}

Cada uno de los forjados, que constituye una planta, fue estudiado con todo detalle, discretizándose como estructura espacial, en la que estaban integrados los pilares y en la que se tuvo en cuenta la existencia de huecos y aligeramientos. El efecto del pretensado fue considerado con todo rigor, ya que el sistema informático empleado permitía introducir en el modelo estructural y en su posición exacta, cada familia de tendones mediante un conjunto de cargas equivalentes en el espacio, deducidas de la geometría real, con sus cambios de curvatura, pérdidas de rozamiento, introducción de cuñas de anclaje, etc. Con ello, los efectos debidos al acortamiento elástico como consecuencia de la compresión del pretensado, quedaban integrados dentro del proceso de cálculo.

El procedimiento empleado permitió determinar la distribución más apropiada del acero activo en fase

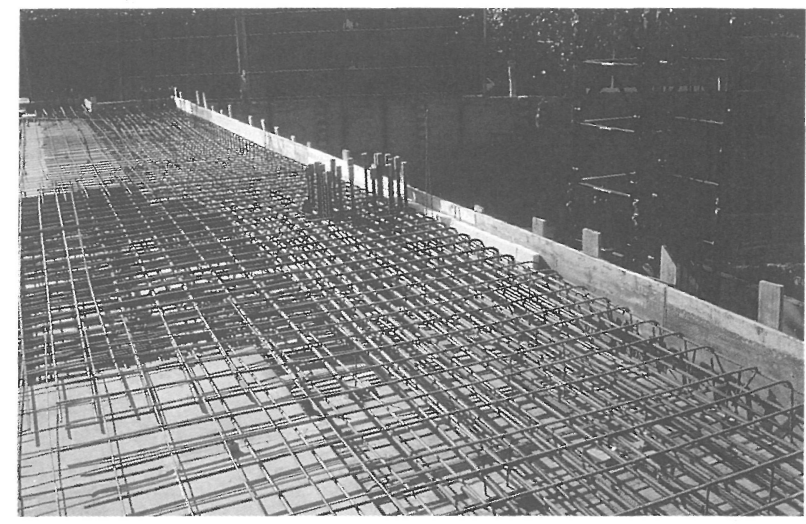

Fig. 15.

de proyecto, ya que durante todo el proceso de cálculo se dispuso de una detallada información en cualquier punto de la estructura (esfuerzos unitarios en losa de forjado, tensiones longitudinales en cualquier dirección, desplazamientos, esfuerzos resultantes en pilares, etc.). El método utilizado hizo posible eliminar el riesgo de las consecuencias de esfuerzos parásitos producidos en las inmediaciones de puntos singulares, tales como encuentros de pilares con las losas, bordes de grandes huecos, esquinas, cambios bruscos de espesor, etc. La adición de armadura pasiva se estimó necesaria en dichos puntos (Figs. 14 y 15), debido al grado de incertidumbre que normalmente implican los resultados derivados de una modelización estructural, por muy detallada que sea, si bien pudo realizarse con relativa tranquilidad, habida cuenta de tener el control, en lo fundamental, del estado tensional de la totalidad de la estructura.

Una de las consecuencias de la labor realizada en lo referente al análisis estructural, fue la optimización de la cantidad de acero de pretensado puesto en obra. A pesar de la gran concentración de armadura activa, existente en puntos singulares, el valor medio resultante fue moderado, ya que representó $7,8 \mathrm{~kg}$ por metro cuadrado de forjado.

El comportamiento de la estructura construida respondió a las previsiones deducidas del cálculo. Posteriormente al descimbrado de cada planta se procedió a una cuidadosa inspección de las superficies de hormigón, no habiéndose observado fisura alguna de carácter estructural.

\subsection{Construcción}

La profusión de especificaciones, imprescindibles por la complejidad de la obra, tuvo por resultado la 


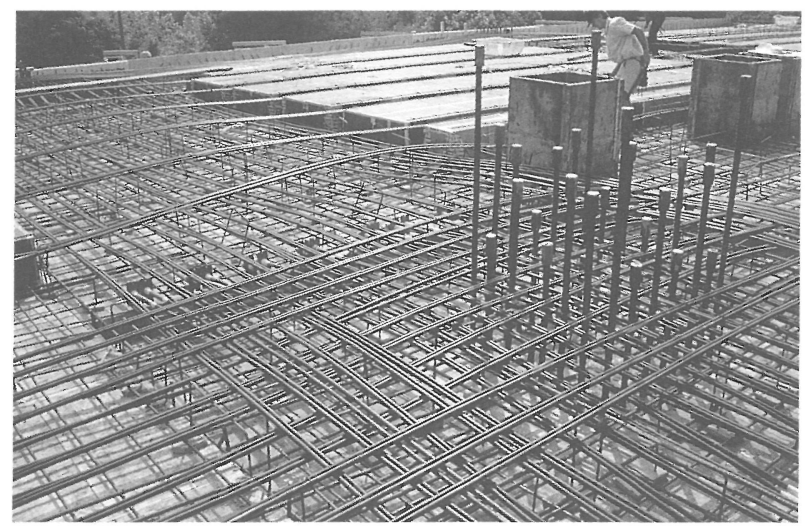

Fig. 16.

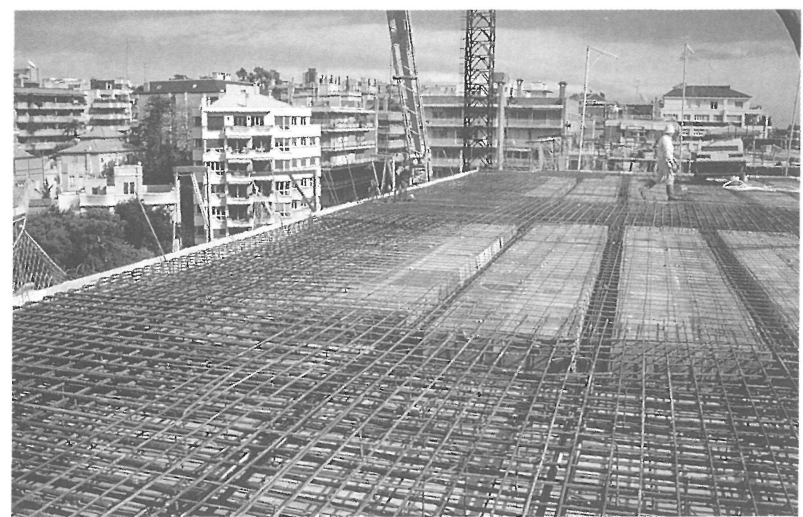

Fig. 17.

simplificación de las operaciones fundamentales y la reducción del plazo de ejecución, en relación con las previsiones iniciales.

En lo referente a la colocación de tendones para el postesado, se elaboró previamente un detallado estudio, determinándose el orden de puesta en obra para evitar interferencias y enhebrados de dificultosa ejecución (Fig. 16). Los tendones, con sus correspondientes anclajes, se formaron en taller y para cada planta se agruparon en diversas familias, identificadas con señales de diversos colores. Gracias a la labor de preparación, junto con el grado de dedicación y conocimiento de los operarios, la colocación de los tendones de cada planta, en número superior a los 300 y con un ajuste geométrico sometido a una estricta tolerancia, se llevó a cabo durante una jornada de trabajo.

En cuanto al conjunto de la obra se consiguió, asimismo, un rendimiento digno de mención. Se efec-

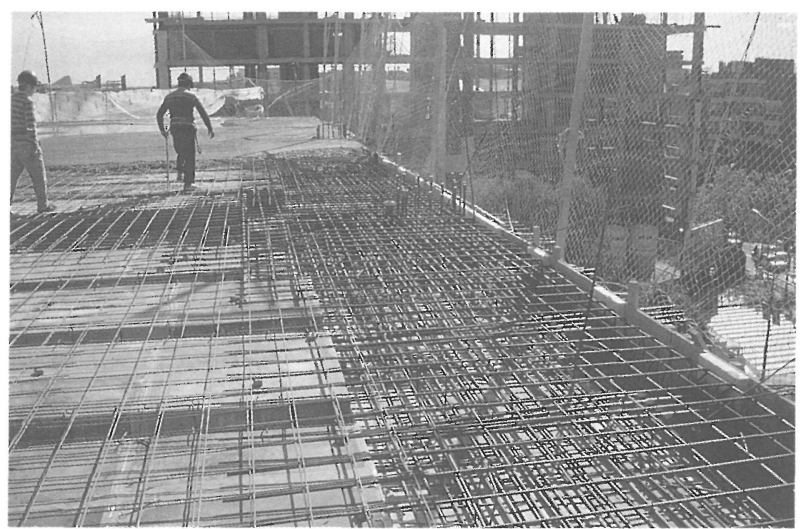

Fig. 18.

tuó el ciclo de construcción completo de cada uno de los forjados de ambas torres (de $1.400 \mathrm{~m}^{2}$ de superficie) en el intervalo de tiempo de nueve días naturales; es decir, con un ritmo real de avance, de $2.800 \mathrm{~m}^{2}$ de forjado completo cada nueve días (las Figs. 17 y 18 muestran imágenes del hormigonado de un forjado).

\subsection{Conclusiones del empleo del postesado no adberente en edificación}

La ejecución de esta obra, caracterizada por la magnitud de las luces y sobrecargas, junto con la disposición de grandes huecos, permite deducir la conveniencia de adoptar el postesado con tendones no adherentes. El sistema ofrece la posibilidad de resolver satisfactoriamente estructuras de cierta importancia que requieran crear espacios diáfanos, sin los inconvenientes derivados de la flexibilidad de los forjados y la necesidad de precisar grandes 
espesores de losas, o jácenas de gran canto. A ello hay que añadir como ventajas fundamentales, la economía obtenida de la cantidad de materiales estructurales a disponer y las posibilidades de rapidez de ejecución.

La tecnología, suficientemente desarrollada, apoyada por las posibilidades del análisis estructural con los medios de que se dispone hoy en día, permite vislumbrar una perspectiva de futuro en España, donde esperamos haya ocasión de nuevas realizaciones en los próximos años.

\subsection{Realización del proyecto y obra}

El Proyecto del edificio ha sido desarrollado en el Estudio PV-13, de los Arquitectos Íñigo Ortiz y Enrique León.

La parte de Proyecto correspondiente a la estructura ha sido realizada en el Estudio de Ingeniería y Proyectos (EIPSA), por los autores del presente artículo, así como la asistencia técnica durante el desarrollo de la obra.

\section{Constructor: OCP. Sistema de postesado no adherente: MeKano 4.}
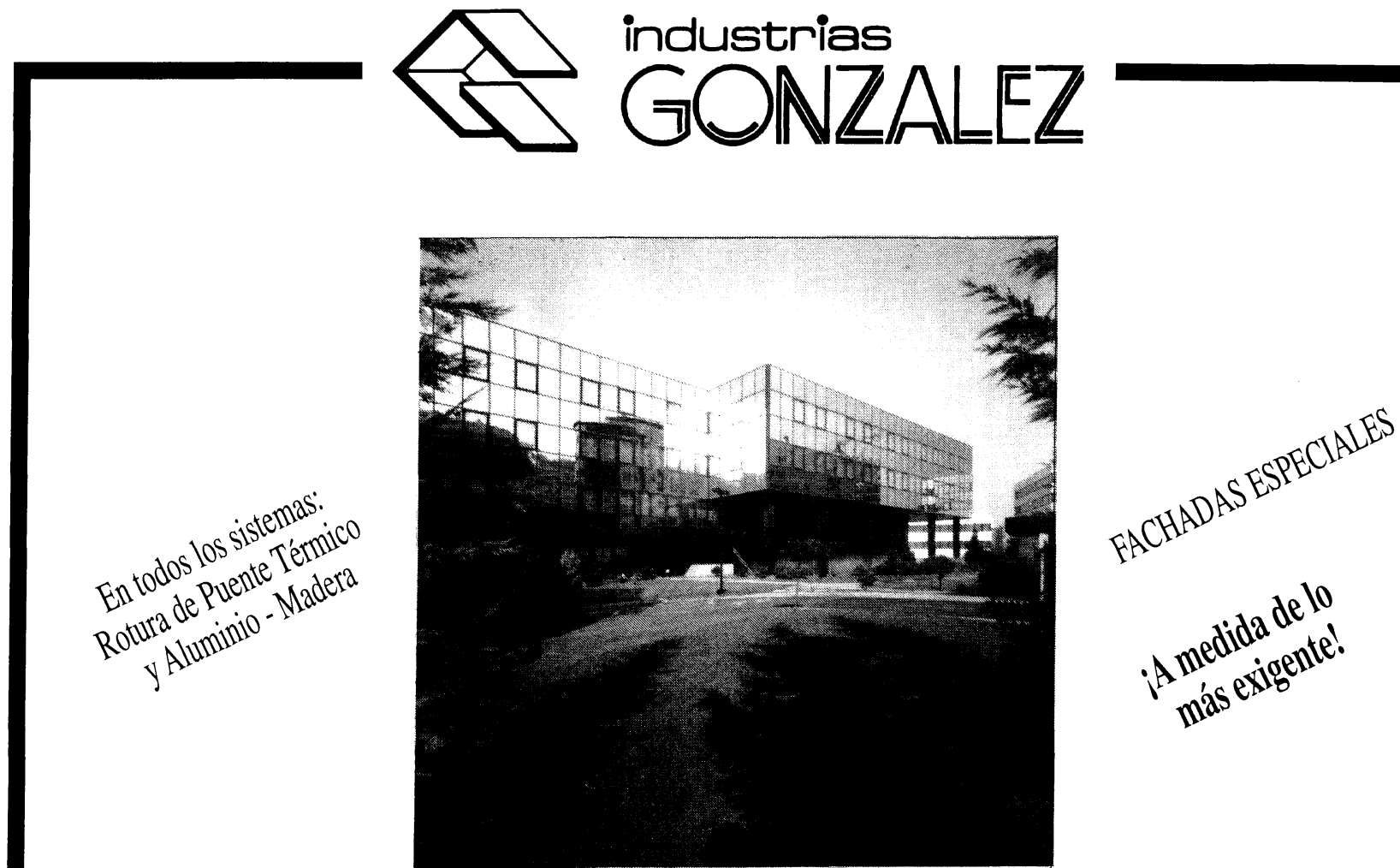

CERRAMIENTO EXTERIOR DE ALUMINIO

FABRICA: Carragoso, 53• Apartado 41• Teléfs. 780070 - 780850 • Fax $780774 \bullet 36500$ LALIN (Pontevedra) 